\title{
The control of insect-borne disease through recombinant DNA technology
}

\author{
PAUL EGGLESTON \\ Wolfson Unit of Molecular Genetics, Liverpool School of Tropical Medicine, Pembroke Place, Liverpool L3 $50 A$, UK
}

\section{Introduction}

Disease transmitted by insects, and particularly by mosquitoes, pose some of the most serious health care problems in the World today. Well known examples include the transmission of malaria, filariasis and arboviruses by anopheline, culecine and aedine mosquitoes, respectively. The symptoms of malaria result from infection with protozoan parasites of the genus Plasmodium, commonly transmitted by Anopheles gambiae. Some 500 million people are estimated to be affected each year, with approximately 3 million deaths (Sturchler, 1989). Moreover, the incidence of infection appears to be increasing, rather than being brought under control. The mosquito, Culex quinquefasciatus, is the principal vector of Bancroftian filariasis in a number of tropical regions. This severe and debilitating disease results from infection with the nematode worm Wuchereria bancrofti which, in many cases, can completely block the lymphatic system to cause elephantiasis (Sasa, 1976). Each year, some 250 million people are believed to be infected with this parasite. Aedes aegypti, commonly known as the yellow fever mosquito, is the primary urban vector of a number of arboviruses (so called because they are arthropod borne) including yellow fever, dengue, dengue haemorrhagic fever (DHF), Japanese encephalitis and La Crosse fever. These acute diseases also affect millions of people and result in substantial mortality. Outbreaks tend to be sporadic with urban epidemics arising frequently, making DHF a major cause of hospitalization and death among children in many Asian countries (Rudnick, 1967). DHF results from serial infection with two of the four serotypes of the dengue virus. The presence of antibodies to one serotype appears to facilitate the invasion of macro- phages by the second dengue serotype, leading to subcutaneous and visceral organ haemorrhage. In some cases, children are born carrying maternal antibodies to a Dengue virus with the result that their first infection may lead directly to the more serious symptoms of DHF.

For a variety of reasons we, and others, have chosen to work with the mosquito Ae.aegypti. The increasing importance of this insect as an urban disease vector, together with its suitability for laboratory research and potential for genetical studies, has resulted in it being considered as a 'promising subject for genetic control' (Knipling et al., 1968). More recently, it has become the focus of research into mosquito molecular biology. It has a simple karyotype $(2 n=6)$, consisting of three distinguishable chromosome pairs each of which has been extensively mapped. The species therefore has a rich source of genetic diversity. It is easily maintained and manipulated in the insectary and is amenable to mass rearing techniques. Females, the only sex that bites man, lay fertilized eggs after receiving a blood meal from an anaesthetized guinea-pig. These embryos can be dried and stored for up to three months before being stimulated to hatch in water. As a result of these attributes, more is known of the genetics and ecology of this insect than for any other medically or commercially important insect.

Under natural conditions, Ae.aegypti is widely distributed throughout the warmer countries $\left(45^{\circ} \mathrm{N}-35^{\circ} \mathrm{S}\right)$ and is frequently involved in urban arboviral epidemics in Africa, Asia, Central and South America, the Caribbean and Southern USA. It has developed a particularly close association with man, travelling and colonizing with him and breeding readily wherever rain water collects, frequently in water containers, refuse and discarded car tyres. 
Research over the last century has increased our understanding of the complex relationship between insect vectors, pathogens causing the disease and the human host. One way of interrupting this cycle is to suppress the insect, which acts as the vector, and a number of techniques have been tried. These include chemical control through insecticides and larvicides, biological control through natural predators or toxins, such as that produced by Bacillus thuringiensis, environmental control through the removal of breeding sites and the raising of public awareness and genetic control through the mass release of sterile males. Another genetic approach, which may become a possibility through the advent of recombinant DNA technology, would be to compromise the vectorial capacity of the insect.

Despite the current trend towards integrated pest management, in which several approaches are used in combination to suppress insect populations, the main emphasis for the control of Ae.aegypti is on the elimination of breeding sites and the application of chemical agents. There are now considerable problems associated with the use of synthetic insecticides. The most important of these is the seemingly inevitable evolution of resistance and there are now populations which are multiply resistant to all four classes of insecticidal compound (organophosphates, organochlorines, carbamates and pyrethroids). Coupled with this is the high cost of developing and registering new insecticidal variants, increasing legislation over their use and growing environmental awareness over their toxic residues. Genetic control, through the mass release of sterile Ae.aegypti males, has also been attempted but with little success (Grover, 1985). This is now thought to be due to the poor competitive mating ability of treated males following chemical or radiological sterilization. Less traumatic sterilization treatments may increase the effectiveness of such a tragedy, as demonstrated by the successful eradication of the screw-worm fly from the Southern USA (Krafsur et al., 1987). However, such autocidal strategies of population control often prove prohibitively expensive as they involve the repeated mass release of treated males (McDonald et al., 1977).

Recent advances in recombinant DNA technology, and the generation of transgenic insects, herald a new era of genetic control. We anticipate that germ line manipulation of medically or commercially important insects may provide a useful alternative strategy. Modifications to the genome would be inherited by subsequent generations, potentially removing the need for frequent mass releases. The ability to introduce and express foreign genes and/or disrupt existing gene functions may lead to the development of a variety of control strategies. It may be possible, for example, to produce pathogen-refractory strains, to reduce reproductive potential or vector competence, or to increase vector susceptibility to existing control measures. Such a facility would have two immediate advantages over conventional genetic techniques. One is the potential to exploit genes across species barriers and the other is the ability to introduce defined DNA sequences without the genome disruption which accompanies a conventional cross. Clearly, the usefulness of genetic manipulation as an approach to the control of insectborne disease has first to be assessed and there will be many practical, and perhaps ethical, difficulties to overcome. Nevertheless, transgenic technology clearly has considerable potential both for the furtherance of our understanding of mosquito molecular biology and for the development of novel genetic control strategies.

\section{The requirements for genetic manipulation}

Despite the dramatic advances made recently with respect to genome manipulation in Drosophila melanogaster, there is an urgent need for a much greater understanding of the molecular biology of mosquito disease carriers. This must include an analysis of the complexity and organization of their genomes and an understanding of the distribution of coding and repetitive sequences. These details form an integral part of the design and interpretation of cloning and hybridization experiments. In addition, the exploitation of transgenic technology requires methods for the introduction of DNA both into living mosquitoes and into cultured cells. Ideally this will involve a transformation vector which is capable of directing efficient and stable integration into the chromosomes of the recipient. The introduced DNA has not only to be expressed but should also carry a selectable marker for the identification of transformed individuals or cells. Ultimately, there will be a need to study alternative promoter and enhancer sequences so that the spatial and temporal expression of the introduced DNA may be controlled. Finally, none of this will be of any relevance unless appropriate genetic target systems can be identified, cloned and characterized at the molecular level.

\section{Genome characterization}

The DNA of all higher eukaryotes is conveniently subdivided into three components, namely highly repetitive, moderately repetitive and unique or single copy sequences. In our laboratory, Julian Crampton, Ann Warren and Teresa Knapp have used reassociation kinetics to determine the total genome size and complexity and the individual complexities of the three 
components for Ae.aegypti DNA (Crampton et al., 1990a). Such experiments involve denaturing the DNA and measuring the reassociation of complementary strands over time. Generally, highly repetitive sequences will reassociate most quickly because there are so many copies whereas unique sequences will reassociate more slowly. The complexities of these components and of the total genome are determined by comparing the reassociation values with that of a single copy sequence of known complexity, namely $E$. coli DNA. Comparison of the genomic DNA from larvae and cells (Table 1) shows that the latter exhibits a twofold increase in total genome size. This may reflect aneuploidy in the cultured cells (Freshney, 1986) or elevated levels of replicative transposition of endogenous transposable genetic elements (part of the moderately repetitive component) as found in $D$. melanogaster following the culture and passage of embryonic cells (Potter et al., 1979). Clearly, the normal constraints of differentiation and homeostasis, which regulate such functions in the living insect, are absent in the cell line.

The data in Table 1 show that the Aeaegypti genome is both large and complex, being five times larger than the genome of Drosophila or, to place it in context, one-third the size of the human genome. Of equal importance, however, is the way in which the different components are distributed throughout the genome. Black \& Rai (1988) suggest that the existence of two basic organization patterns throughout all

Table 1 Sequence organization of Aedes aegypti larval and cell line DNA (data kindly provided by Julian Crampton, Ann Warren and Teresa Knapp)

\begin{tabular}{lll}
\hline & $\begin{array}{l}\text { Bangkok } \\
\text { larvae }\end{array}$ & $\begin{array}{l}\text { Mos 20 } \\
\text { cell line }\end{array}$ \\
\hline Total genome size $(\mathrm{bp})$ & $8.0 \times 10^{8}$ & $1.5 \times 10^{9}$ \\
Picograms DNA/cell & 0.83 & 1.5 \\
Proportion of genome & & - \\
HR DNA & 0.2 & 0.13 \\
MR DNA & 0.2 & 0.24 \\
SC DNA & 0.6 & 0.64 \\
Total base pairs & & \\
HR DNA & $1.6 \times 10^{8}$ & $1.4 \times 10^{8}$ \\
MR DNA & $1.6 \times 10^{8}$ & $2.6 \times 10^{8}$ \\
SC DNA & $4.8 \times 10^{8}$ & $1.1 \times 10^{9}$ \\
Average reiteration frequency & & \\
HR DNA & $10^{5}-10^{6}$ & $10^{5}$ \\
MR DNA & $10^{3}$ & $10^{3}$ \\
SC DNA & 1 & 1 \\
\hline
\end{tabular}

higher eukaryotes is indicative of a set of rules governing the establishment and spread of repetitive elements. The first pattern is known as short period interspersion (SPI), in which 1-2 kb segments of single copy sequence alternate regularly with short $(0.2-0.6 \mathrm{~kb})$ or moderately long (1-4 kb) repetitive sequences. This pattern is characteristic of the majority of animal species. The second pattern, long period interspersion (LPI), is characterized by long (5-6 kb) repetitive sequences alternating with very long $(>13 \mathrm{~kb})$ uninterrupted stretches of unique-sequence DNA. Clearly, the evolution of such organization is an interesting phenomenon in itself and the family Culicidae (to which the mosquitoes belong) may be of particular interest. According to Black \& Rai (1988), this is the only family so far shown to contain species that exhibit both patterns of organization and it may, therefore, explain how the transition from one to the other occurs.

Reassociation kinetics, together with screening experiments in which Ae.aegypti genomic libraries were hybridized with total genomic and rDNA probes, show clearly that this species has a SPI pattern. Thus, almost every clone isolated from genomic libraries will contain repetitive DNA and this may well mask the hybridization characteristics of sequences of interest (particularly single copy sequences) when using complex genomic probes. This is in direct contrast to an organism like Drosophila, which displays a LPI pattern, where cloned sequences are more likely to consist entirely of either repetitive or unique DNA. In insect studies to date, Anopheles quadrimaculatus, Drosophila melanogaster, Apis mellifera, Sarcophaga bullata and Chironomous tentans have been shown to exhibit the LPI pattern. Conversely, Aedes aegypti, Culex pipiens, Aedes triseriatus, Aedes albopictus, Lucilia cuprina, Musca domestica, Bombyx mori and Antherea pernyi exhibit the SPI pattern (Black \& Rai, 1988; Crampton et al., 1990 and T. Howells personal communication). There appears to be no simple relationship between genome size and organization.

Because of the SPI pattern of genome organization in Ae.aegypti we have made considerable use of short synthetic oligonucleotide probes and we have become increasingly aware of variations in codon usage between different organisms. An analysis of related maltase genes in D. melanogaster (Snyder \& Davidson, 1983), Ae.aegypti (James et al., 1989) and Saccharomyces carlsbergensis (Aota et al., 1988) shows some typical differences. Considering the proportions of each of the four nucleotides at each of the three codon bases, over $90 \%$ of the total variance resides at the third base. For this base Drosophila shows a clear preference for the nucleotides $\mathrm{G}$ or $\mathrm{C}(72 \%)$ whereas 
Saccharomyces is biased towards A or T $(61 \%)$. No clear preference is seen for the mosquito gene although there is a slight excess of the nucleotides $G$ and $C$. Given these differences, it is clear that Drosophila sequences may not always make suitable probes and this is one reason to support the use of oligonucleotides. However, oligonucleotides used to probe Ae.aegypti libraries should ideally be synthesized as mixed populations incorporating variable third base nucleotides.

\section{The development of transgenic technology}

Germ-line transformation of insects and transfection of cultured cells requires the establishment of appropriate techniques for the introduction of a eukaryotic DNA transformation vector. This should be capable of directing the efficient and stable integration of DNA sequences into the chromosomes of the recipient, as well as providing some means for the identification of transformants. The transposable genetic $\mathrm{P}$ element from $D$. melanogaster has been used in a variety of insect species in an attempt to create transgenic individuals and appropriate transformation vector constructs are now used as a routine research tool in Drosophila.

P elements (Engels, 1988) are a distinct class of transposable element discovered when a syndrome of correlated genetic traits (hybrid dysgenesis) occurred if male flies from $\mathrm{P}$ strains (normally from wild populations) were mated with $\mathrm{M}$ strain females (normally from laboratory populations). A series of abnormalities resulted, which included high rates of mutation, male recombination, chromosomal rearrangements, sterility, abnormal germline development and meiotic drive. Genetic observations showed that a number of these mutations were unstable and had high reversion frequencies, all of which led to the hypothesis that $\mathrm{P}$ strains carry a family of transposable sequences called $\mathrm{P}$ elements. The abnormalities only arise in hybrids because of the absence of a repressive factor which, although not fully understood, is normally found in $\mathrm{P}$ strains. It is now thought that $\mathrm{P}$ factors have arisen in wild Drosophila only within the past 30 years. The $\mathrm{P}$ element family consists of intact elements $(2.9 \mathrm{~kb})$ and a heterogeneous group of smaller elements $(0.5-2.5$ $\mathrm{kb})$ which are derived from the intact element by internal deletion. The intact element encodes a transposase enzyme which catalyses excision and transposition. The smaller elements lack this function and are incapable of transposing themselves. They can, however, transpose when supplied with functional transposase in trans by an intact element. All P elements carry perfect $31 \mathrm{bp}$ inverted repeat sequences at their termini that are absolutely required for excision and transposition. This process results in an 8 bp duplication at the target site. P elements are capable of precise excision which leaves behind a single copy of the $8 \mathrm{bp}$ sequence. More frequently they will excise imprecisely leaving part of the element behind or removing flanking sequences.

The pUChsneo transformation vector (Fig. 1a) carries only the inverted terminal repeats of the $\mathrm{P}$ element. Between these termini it has been engineered to carry an antibiotic resistance gene, as a marker for the identification of transformed individuals, and a site where foreign DNA can be ligated. The inverted repeats are joined by $500 \mathrm{bp}$ from the white locus of Drosophila, which may function as a hybridization probe to distinguish random integration events from precise P-mediated transposition (Miller et al., 1987). The latter would involve only those sequences within, and including, the inverted terminal repeats of the $\mathrm{P}$ element, but excluding the white locus DNA. In our experience however, this diagnostic feature is limited, perhaps because of a low frequency of precise excisions. The site of integration of foreign DNA within the genome, rather than the precision of the mechanism, is likely to be of greater importance to normal gene expression.

Transposition of vector sequences and integration into the genome will only be mediated by the terminal repeats if they are supplied with a functional transposase. A helper plasmid, pUChs $\pi(\Delta 2-3)$, which carries the entire $\mathrm{P}$ element transposase coding region (Fig. 1b), is therefore introduced with the transformation vector. Although the helper carries most of the $\mathrm{P}$ element sequences, it is unable to transpose and integrate into the genome because of a specific deletion in one of its terminal repeats (Steller \& Pirrotta, 1985). The sole function of the helper is therefore to supply transposase and, in Drosophila, further injections of helper have been used to switch transposition on in those generations following the initial transformation.

Normally, P element transposition occurs only in the germ line of Drosophila because the intron between open reading frames 2 and 3 of the coding sequence is not removed in somatic tissue. However, transposase expression from constructs which have been modified in vitro to remove this intron (Fig. 1b), does allow transposition in somatic tissue. There is now good evidence that similar processing problems prevent normal $\mathrm{P}$ element transposition in non-drosophilids (O'Brochta \& Handler, 1988) although excision has been observed in mammalian and yeast cells (Rio et al., 1988). If there is a requirement for non-P elementencoded proteins to achieve transposition in Drosophila, then the distribution of homologous genes in related genera may explain the divergent results 
(a)

pUChsneo transformation vector

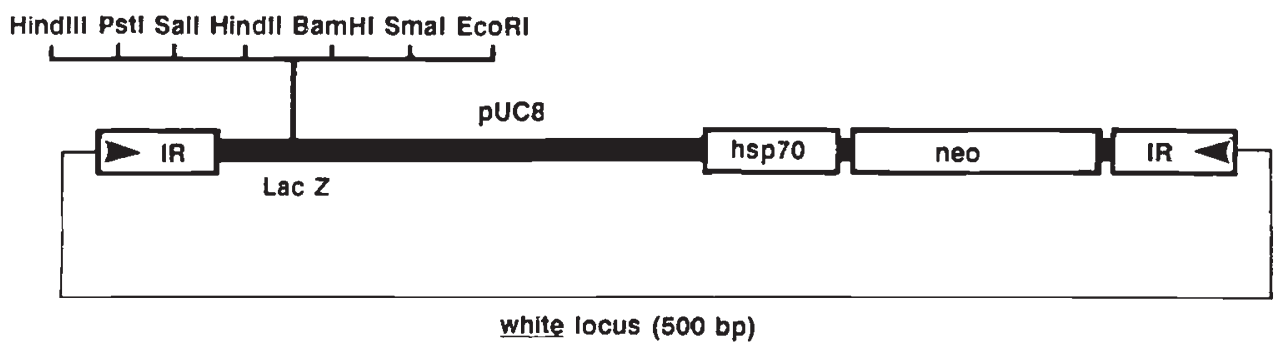

(b)

pUChs $\pi(\Delta$ 2-3) helper element

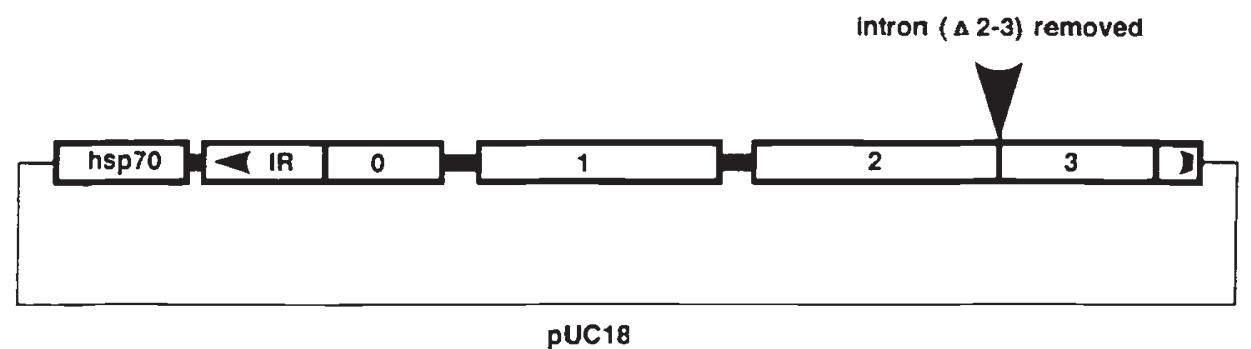

Fig. 1 (a) The pUChsneo transformation vector carries the bacterial neomycin resistance gene derived from $T n 5$ driven by the Drosophila heat shock promoter, hsp70. The multiple cloning site from pUC8 allows foreign DNA to be incorporated between the inverted terminal repeats of the P transposable element (IR). The repeats are joined by $500 \mathrm{bp}$ of white locus DNA from Drosophila.(b) The pUChs $\pi(\Delta 2-3)$ helper plasmid carries the entire $P$ transposase coding sequence (exons $0,1,2$ and 3 ), which is transcribed under the control of the Drosophila heat shock promoter, hsp70. The sequence has been modified in vitro to remove the intron between open reading frames 2 and 3 in order to overcome the germ line specificity observed in Drosophila, and cloned into pUC18. One of the inverted terminal repeats of the $\mathrm{P}$ transposable element carries a $23 \mathrm{bp}$ deletion which prevents the integration of the helper into the recipient genome.

obtained in other insects. In our laboratory, Alison Morris has detected the integration of pUChsneo sequences in Ae.aegypti (Morris et al., 1989) and similar results have been obtained in An.gambiae (Miller et al., 1987) and Ae.triseriatus (McGrane et al., 1988). Although these events did not result from normal $\mathrm{P}$ element transposition, some functional role of the $\mathrm{P}$ sequences cannot be excluded. This is particularly true as similar experiments in Lucilia cuprina ( $\mathrm{T}$. Howells, personal communication) and Ceratitis capitata (M. Ashburner, personal communication) have failed to produce any integration of vector sequences. Research in other laboratories is now being directed towards the identification of these accessory Drosophila proteins and the cloning of the genes involved may facilitate high efficiency $P$ transposition in nondrosophilids.

\section{Micro-injection of Aedes aegypti embryos}

Unlike that of Drosophila, the rigid, opaque endochorion of the mosquito embryo cannot be removed, and the embryos are extremely sensitive to desiccation. In our laboratory, however, Alison Morris and Iain Comley have shown that glass capillaries with tips of $100-300 \mu \mathrm{m} \times 4-10 \mu \mathrm{m}$ can be used to puncture the rigid endochorion without tearing it and deliver the DNA solution without damage to the embryo (Fig. 2a,b). The slightly viscous DNA solution cannot be expelled manually from such a fine needle and is there- 


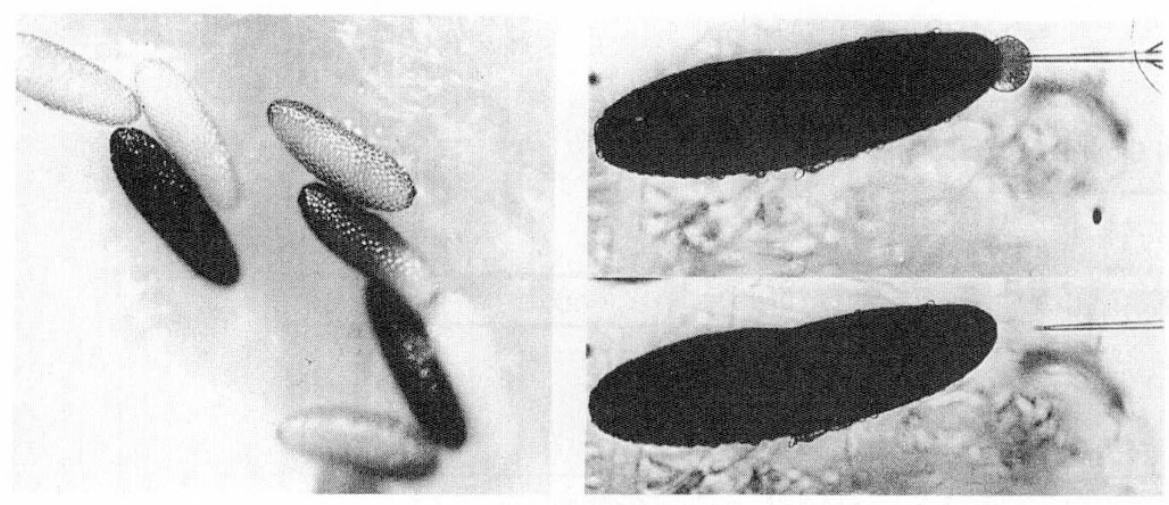

Fig. 2 (a) Early Aedes aegypti embryos at various stages of development. The embryos are white when laid by the female mosquito but gradually darken to become black after about $4 \mathrm{~h}$. Translucent grey embryos, under $2 \mathrm{~h}$ old, are ideal for microinjection. (Scale $\times 30$.) (b) Microinjection of Aedes aegypti embryos. Occasionally cytoplasm is released if the embryo has not been correctly desiccated prior to the injection of DNA solution. This loss is tolerated by the insect provided that it is not excessive. (Scale $\times 80$.) fore injected by means of a two-phase nitrogen supply. The lower pressure prevents backflow and the higher pressure delivers 160-800 pl of DNA solution (corresponding to $1-5 \%$ of the embryo volume) into the posterior pole of the embryo at the syncitial blastoderm stage, before cell partitioning occurs. This is where the pole cells, which are the germ line primordia, develop. Experience from Drosophila suggests that injection of DNA close to the site of pole cell formation is not critical to germ line incorporation, but the timing is clearly important if the DNA is to be taken up by the developing germ line cells. All our injections are normally completed within two hours of oviposition. After injection, the embryos are covered with a watersaturated halocarbon oil, which permits the normal uptake of water until they are returned to standard insectary conditions. In this way transformation vector sequences have been introduced into the embryos, with survival rates comparable to those obtained with Drosophila (Spradiling \& Rubin, 1982).

The basis of the experimental design is shown in Fig. 3 and the molecular nature of any transformation events is determined by DNA analysis using radioactively labelled transformation vector DNA to probe Southern blots of genomic DNA extracted from the putative transformants and their progeny (Miller et al., 1987; Morris et al., 1989). Intact vector P elements have been detected in 5-10 per cent of adults that have developed from injected embryos $\left(\mathrm{G}_{0}\right)$, confirming that the introduced DNA is not immediately broken down by the mosquito. Furthermore, we have detected the chromosomal integration of vector DNA in several $G_{0}$ individuals. This probably reflects direct incorporation into a proportion of the somatic cell nuclei because it is only in the following generation $\left(G_{1}\right)$ that we might expect a germ-line integration event to have been transmitted to every nucleus. More promisingly, vector DNA has been identified in the chromosomes of the $G_{1}$ and $G_{2}$ progeny of injected embryos, which suggests that integration has occurred in the germ-line of the mosquito and that this DNA shows normal Mendelian inheritance. Some of these events, however, appear to be unstable from one generation to the next and this phenomenon, together with the molecular basis of the transformation events is currently being investigated.

\section{Transformation of Aedes aegypti cells in culture}

The transfection of cells in culture can give many insights into the molecular biology of an organism. The simple, controlled environment of cultured cells allows one to follow the expression of cloned genes, and so delineate promoter and enhancer sequences. Genetically manipulated cell cultures are also able to overproduce specific proteins which facilitates their isolation and purification.

In our laboratory, Gareth Lycett has used cultured mosquito cells to examine different transfection vectors and to help establish a suitable system for germ-line transformation of Ae.aegypti (Lycett, 1990). Initial experiments have introduced $\mathrm{P}$ element vector and helper constructs into several cell lines by a variety of techniques devised to generate transient cell membrane pores, including calcium phosphate precipitation (Wigler et al., 1977), dextran sulphate (Lopata et al., 1984) polybrene (Durbin \& Fallon, 1985), electroporation (Chu et al., 1987) and lipofection (Felgner et al., 1987). Much of this work has concentrated on the immortal Mos 20 fibroblast cell line which was derived from minced, trypsinized, neonate larvae of the Ae.aegypti London strain in 1969. Polybrene and electroporation-mediated transfection have proved to be most successful for these cells, producing approximately 30 and 4000 transformants per $10^{6}$ cells, respectively. DNA can be isolated from transformed cell clones and Southern blots probed with radiolabelled transformation vector sequences to analyse individual transformation events. In our experience, 


\section{EXPERIMENTAL DESIGN}
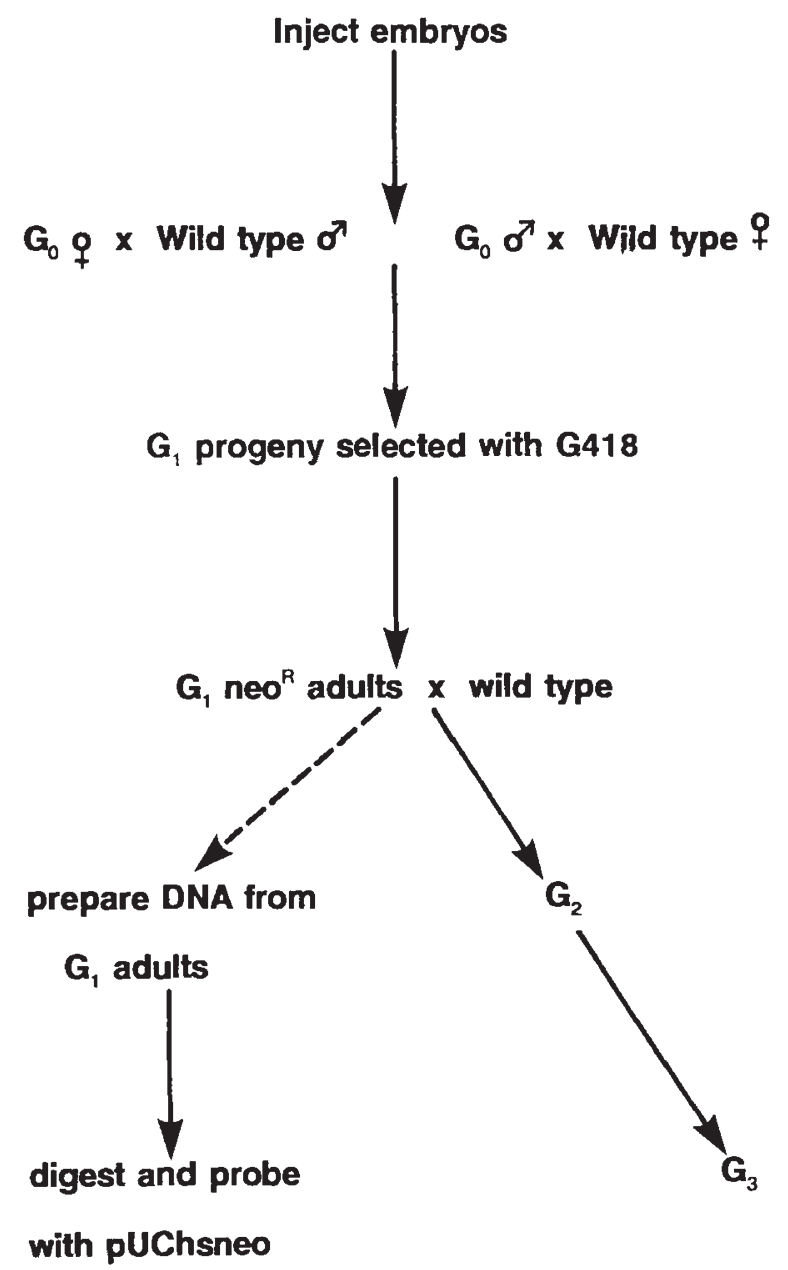

Fig. 3 Summary of the experimental design employed to generate transgenic Aedes aegypti mosquitoes. $\mathrm{G}_{0}$ adults develop from the injected embryos and are mated to wild type individuals. The resulting $G_{1}$ progeny are subjected to selection with the antibiotic G418 and transformed adults mated to wild type to form the $G_{2}$ generation. Subsequent generations are produced in the same way. DNA is extracted from individual adults of each generation, to test whether the foreign DNA has been inherited, once the survival of the following generation has been assured.

these fall into two classes. There are those in which vector sequences have integrated into chromosomal DNA (although these do not correspond to normal $\mathrm{P}$ element transpositions) and those which transiently express resistance from free vector plasmids. Transient expression has been detected for more than 6 weeks, which suggests that the vector plasmid may replicate within the mosquito cells. Current research is being directed towards an analysis of the molecular nature of these transformation events.

\section{The future of mosquito genome manipulation}

Although we can readily generate germ-line integration events in Ae.aegypti it is clear that these do not involve normal $\mathrm{P}$ element transposition. The absence of this controlled mobility poses certain limitations, for example with respect to transposon tagging. Research elsewhere concentrates on the precise mechanism of $\mathrm{P}$ transposition and may, ultimately, isolate the genetic factors involved. Presumably, these could then be incorporated into $\mathrm{P}$ constructs to form the 'universal vectors' originally envisaged. At the same time, we are aware of the possibility that $\mathrm{P}$ elements may never function as efficient transposition-mediated transformation vectors in non-drosophilids. To this end we are investigating a number of alternative transformation vector systems which may improve the overall efficiency. These are all based on transposable elements and include the use of fully processed cDNA copies of both the Drosophila $\mathrm{P}$ element and the Ac and Spm elements from Zea mays. The latter have been shown to transpose actively in a number of evolutionary disparate organisms and may prove to act autonomously in mosquitoes (Kunze \& Starlinger, 1989). By using cDNA clones it is hoped to overcome the problems of transposase processing seen with existing constructs. We are also actively searching for endogenous transposable elements which may yet prove to be the most suitable transformation vectors. In addition, we feel that there is a need for improved selectable marker systems for the identification of transformed individuals and cells. A number of research laboratories have now reported problems with the existing neomycin resistance technique. In our experience, the main problem is the low activity of the neomycin phosphotransferase produced by the Tn5 neo gene in the transformation vector. In addition, Ae.aegypti cells appear to have a tendency to retain intact vector plasmids which do not integrate into the recipient chromosomes but which transiently express antibiotic resistance. Gareth Lycett is now investigating alternative selectable markers including the Tn903 neomycin resistance gene, which has been reported to show much higher phosphotransferase levels in yeast cells (Langhinrichs et al., 1989), and hygromycin resistance, together with alternative promoters to drive their expression. Ultimately, we would like to have cloned phenotypic markers available for use in transgenic mosquitoes, such as the eye colour mutations 
used routinely in Drosophila. This, however, will have to await the results of cloning experiments currently underway in our laboratory. We also feel that it is important to use our existing procedures to begin to analyse the control of gene expression in transgenic mosquitoes. To this end we are using $\beta$-galactosidase and CAT reporter gene fusions both to investigate the functional expression of heterologous proteins and as a test bed for the isolation of mosquito promoter sequences. These will ultimately be necessary to control the temporal and spatial expression of introduced genes.

\section{Transposable genetic elements in the mosquito genome}

The isolation of endogenous transposable genetic elements may ultimately prove central to the development of efficient transformation and transposon tagging systems in mosquitoes. Two approaches were initially envisaged for identifying mobile elements in the Ae.aegypti genome. The first was to analyse specific gene systems, in particular the rDNA and the white eye locus, in an attempt to isolate variants of these genes which may have arisen from the insertion of transposons. Most of this work has concentrated on the rDNA which has a repeat unit of $9.0 \mathrm{~kb}$ in Ae.aegypti. Individual rDNA repeats exhibit a high degree of homogeneity with respect to length and the position of restriction enzyme recognition sites. Preliminary mapping data, together with partial digestion experiments, carried out by Kevin Gale, demonstrate that as in all other higher eukaryotes, the rDNA repeats are arranged in a head-to-tail, tandemly repeating manner (Gale \& Crampton, 1989). The estimate of rDNA repeat copy number in Ae.aegypti, obtained by Kevin Gale (approximately 500 copies per haploid genome), is similar to that of 430 copies per haploid genome reported for the closely related species, Ae.albopictus. Ribosomal DNA thus comprises approximately 0.6 per cent of the total Ae.aegypti genome. An analysis of the variation between strains of Ae.aegypti has served to confirm the remarkable homogeneity of the rDNA repeat unit in this insect and mapping shows that what variation there is derives from restriction enzyme site polymorphisms rather than insertions of mobile elements.

The second approach was to identify DNA sequences which share biochemical and structural properties with previously well-defined eukaryotic transposable elements. Of particular interest were the class of transposable element known as retrotransposons, a characteristic feature of which is their representation in the extrachromosomal fraction of the genome. To this end, Ann Warren has isolated extrachromosomal DNA from Ae.aegypti and used cloned extrachromosomal sequences to probe Southern blots of total genomic DNA. One such clone, pX16, displays intraspecific variation in chromosomal distribution and genome copy number, both of which are diagnostic features of transposable elements. Estimates of copy number range from 30 in the Bangkok strain to 600 in the Mos 20 cell line, thus confirming the middle repetitive nature of the $\mathrm{pX} 16$ insert sequence. Further analysis has shown that $\mathrm{pX} 16$ has an RNA representation (as might be expected for a retrotransposon) and partial sequence data shows considerable homology to the consensus sequence in several retroviral and retrotransposon reverse transcriptases. These properties clearly indicate the mobile nature of pX16 and, moreover, its isolation as a circular, extrachromosomal molecule suggests a similarity with retrotransposons rather than non-viral retroposons. Integrated copies of this element are being sought using cosmid libraries and sequence analysis of the terminal regions and adjoining host sequences will detect structural hallmarks, such as long terminal repeats and direct host repeats, flanking the integrated element. This will ultimately allow the unambiguous classification of the pX16 element.

\section{Potential target genes for manipulaton}

Several types of useful target gene can be envisaged. There are those that render populations vulnerable to subsequent control measures (such as insecticide susceptibility, temperature sensitivity or inability to survive diapause); those that interrupt disease transmission by replacing vector with non-vector forms and those that disrupt normal fertility, development or behaviour (Fig. 4). Attempts are now being made to isolate a number of these genes.

Changes in esterase activity are correlated with insecticide resistance in a number of insects and the esterases which are involved in organophosphate resistance in Culex quinquefasciatus have been cloned and characterized, both in this laboratory by Alison Merryweather, and by researchers elsewhere (Mouches et al., 1986; Merryweather et al., 1990). In addition, the Ae.aegypti cytochrome P-450 genes, which are involved in more general detoxification mechanisms, have now been isolated in our laboratory by Roger Gerke Bonet (Bonet et al., 1990).

A number of genes are of particular interest because they are directly implicated in the ability of the insect to transmit parasites. Examples include the filarial susceptibility $\left(\mathrm{f}^{m}\right)$ and Plasmodium susceptibility $(p l s)$ loci of Ae.aegypti. The $\mathrm{f}^{m}$ locus is genetically well 


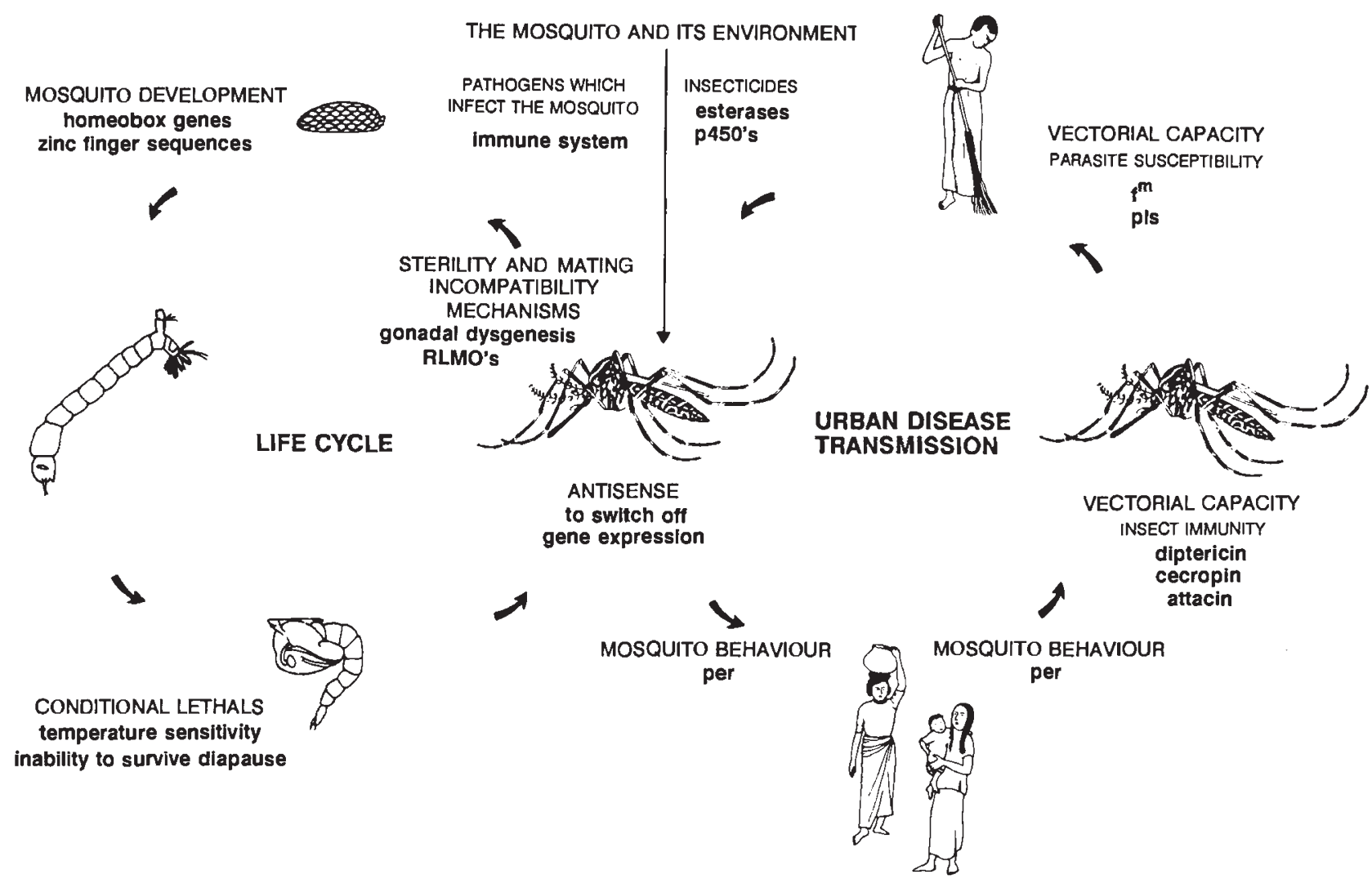

Fig. 4 Schematic representation of a number of genetic target systems and the ways in which they interact with the life cycle and disease transmission cycle of the mosquito, Aedes aegypti. Each target system has been suggested on the basis of two criteria. The first is that the genes involved must have potential for the suppression of mosquito populations or interruption of the disease transmission cycle. Secondly, there must be an accessible procedure by which the relevant genes can be isolated and cloned for analysis. In many cases, this will involve the use of heterologous probes from related insects, although it may demand a more direct approach based on a knowledge of the genetics and biology of the Aedes mosquito itself. Further details of the individual systems are included in the text.

defined and we have good data on its linkage relationships. Refractoriness to infection is due to a partially sex-linked, dominant gene (Macdonald \& Ramachandran, 1965). There is marked variation in the susceptibility of this mosquito to different filarial worms, although all of the alleles concerned map at about the same place on the sex chromosome. We are currently attempting to clone this gene by microdissection from the polytene chromosomes. Sequences isolated in this way will be used to initiate chromosome walks (probably in conjunction with jumping, linking and cosmid libraries) into the region of interest.

For similar reasons we are interested in the genes that code for insect immune proteins, such as the attacins, cecropins and diptericin (Boman et al., 1987). These proteins are induced in response to infection and are likely to be involved in the ability of insects to transmit pathogens. Various sequences of interest have now been isolated in our laboratory by Teresa Knapp, using appropriate synthetic oligonucleotide probes, and these are currently being characterized.

Genes involved in the control of development may provide useful targets for future genetic manipulation. Of particular interest are those sequences thought to exert a controlling influence on the developmental cascade by acting as transcriptional regulators. Examples include the homeobox sequences, first identified in Drosophila (Gehring, 1987) and the so-called zinc finger genes. Oligonucleotide and heterologous genomic probes have been used to clone a number of putative homeobox genes from Ae.aegypti and these are currently being analysed by restriction mapping and sequencing.

The genes involved in controlling other physiological processes may also provide interesting targets for insect control. For instance, the per gene is involved in controlling circadian rhythms in many organisms including insects (Hardin et al., 1990). In Drosophila, 
this gene controls the timing of eclosion and some rhythmic components of courtship song (Reddy et al., 1984). Homologous sequences have now been isolated from Ae.aegypti by Julian Crampton and Teresa Knapp and these will be used to analyse the behavioural role of this gene in the mosquito.

Other potentially useful targets would be sterility or mating incompatibility mechanisms known to have a genetic basis. Examples of particular interest would include the mating incompatibility generated by Rickettsia-like micro-organisms in various insects (Binnington \& Hoffmann, 1989) and the reduced fertility caused by mobile element transposition (for example by P elements) in Drosophila (Engels, 1988).

Finally, we intend to explore the potential of antisense sequences to switch off the normal expression of genes. This can be accomplished by creating a transgenic mosquito such that the antisense strand of the artificial gene, rather than the sense strand, is transcribed (Day, 1989). The resulting antisense mRNA binds to the normal mRNA forming double helices that cannot be translated by the ribosomes.

\section{Transgenic mosquitoes in natural populations}

Now that the technology for the introduction of artifical genes is becoming established, it is necessary to consider the problems likely to be faced in experimental and natural populations. It may well be that such a situation would disrupt the normal adaptive process and therefore be opposed by natural selection. If this is so then some form of drive mechanism may be needed to force the desired gene through the population. This is not an alien concept to those who have worked on the genetic control of insect populations. However, the testing of such mechanisms has been limited as, in reality, they have awaited the advent of recombinant DNA technology to provide the necessary raw material.

Two types of drive mechanism have been suggested. One is meiotic drive, where a given chromosome is transmitted to more than the expected 50 per cent of offspring. Any desirable genes linked to the driven chromosome would eventually approach fixation even with the release of relatively few individuals. There is experimental evidence to support the use of meiotic drive in Ae.aegypti. This mechanism, driven by the $\mathrm{M}^{D}$ locus has been used to force the marker gene re (red eye) into a laboratory cage population (Wood et al., 1977). Interestingly, meiotic drive also occurs during hybrid dysgenesis and it might, therefore, also be possible to exploit this phenomenon by using $\mathrm{P}$ elements in Ae.aegypti. The second type of drive mechanism is the exploitation of genetic traits that reduce heterozygote fitness (Curtis \& Graves, 1988). For example, the gene to be driven could be introduced into a translocation chromosome such that viable and fertile homozygotes were formed, whereas heterozygotes would display reduced fertility or viability. In this way, translocations, pericentric inversions, interracial hybrid sterility, cytoplasmic incompatibility and compound chromosomes all have potential as, in each case, hybrids have reduced fitness. Such mechanisms require larger release numbers because there is no exponential increase in the frequency of the driven chromosome as with meiotic drive. However, fixation of desirable genes would occur more quickly than with meiotic drive because of the reduced fitness of heterozygous combinations. Efficiency could be improved by providing the released individuals with some form of temporary advantage. For example, insecticide resistance could be incorporated into the genome and then insecticide applied (Whitten, 1970). Ideally, the insecticide resistance gene would be fused to the desirable gene and introduced as a unit to prevent disruption of useful combinations by meiotic recombination. The most useful end result of such programmes would be the progressive replacement rather than the eradication of disease-transmitting populations as an emptied ecological niche might be colonized rapidly by the migration of wild types.

Eventually, embryo transformation will provide the raw material to test these proposed drive mechanisms properly in laboratory and natural populations. The possible hazards of releasing this type of manipulated organism are difficult to assess in the absence of experimental evidence and will clearly require regulation. However, the potential of this technology is such that it must be explored, not only in the mosquito, but probably in many medically or commercially important species. Only then can a sensible assessment be made of the balance between likely benefits and potential problems.

\section{Acknowledgements}

The material presented here describes the work of current and former members of the mosquito research group within the Wolfson Unit of Molecular Genetics. I am particularly indebted to Julian Crampton, Ann Warren, Alison Morris, Gareth Lycett, Teresa Knapp, Kevin Gale, Iain Comley, Alison Merryweather and Roger Gerke Bonet for the provision of data and the use of their research results. I am grateful for the financial assistance provided by The Lister Institute of Preventive Medicine, through the award of a Lister Institute Research Fellowship. Additional financial assistance has been provided during the course of this 
research by The Wellcome Trust, MRC, Wolfson Foundation and the University of Liverpool. We are grateful for their support.

\section{References}

AOTA, S., GOJOBORI, T., ISHIBASHI, F., MARUYAMA, T. AND IKEMURA, T. 1988. Codon usage tabulated from the GenBank Genetic Sequence Data. Nucl. Acids. Res., 16, r315-r402.

BINNINGTON, K. C. AND hofFMANN, A. A. 1989. Wolbachia-like organisms and cytoplasmic incompatibility in Drosophila simulans. J. Invert. Pathol., 54, 344-352.

BLACK, W. C. AND RAI, K. s. 1988. Genome evolution in mosquitoes: intraspecific and interspecific variation in repetitive DNA amounts and organisation. Genet. Res. (Camb.), 51, 185-195.

BOMAN, H. G., FAYE, I., GAN, R. et al. 1987. Insect immunity: a gene system for antibacterial proteins. Mem. Inst. Oswaldo Cruz., 82 (Suppl. 3) 115-124.

BONET, R. G., CRAMPTON, J. M. AND TOWNSON, H. (1990) P450 sequences from the mosquito Aedes aegypti. Trans. $R$. Soc. Trop. Med. Hyg., 84, 453.

CHU, G., HAYAKAWA, H. AND BERG, P. 1987. Electroporation for the efficient transfection of mammalian cells with DNA. Nucl. Acids. Res., 15, 1311-1326.

CRAMPTON, J. M., MORRIS, A. C., LYCETT, G. J., WARREN, A. M. AND EgGleston, P. 1990a. Transgenic mosquitoes: a future vector control strategy. Parasitology Today, 6, 31-36.

CRAMPTON, J. M., MORRIS, A. C., LYCETT, G. J., WARREN, A. M. AND EGGLESTON, P. 1990b. Molecular characterisation and genome manipulation of the mosquito, Aedes aegypti. In: Hagedorn, H. H., Hildebrand, J. G., Kidwell, M. G. and Law, J. H. (eds) Molecular Insect Science, Plenum Press, New York, pp. 1-11.

CURTIS, C. F. AND GRAVES, P. M. 1988. Methods for replacement of malaria vector populations. J. Trop. Med. Hyg., 91, 43-48.

DAY, s. 1989. Switching off genes with antisense. New Scientist, 124 (1988), 50-55.

DURBIN, J. E. AND FALLON, A. M. 1985. Transient expression of the chloramphenicol acetyl transferase gene in cultured mosquito cells. Gene, 36173-178.

ENGELS, w. R. 1988. P elements in Drosophila melanogaster. In: Berg, D. and Howe, M. (eds) Mobile DNA, ASM Publications, Washington, D.C., pp. 437-484.

FELGNER, P. L., GADEK, T. R., HOLM, M. et al. 1987. Lipofection: a highly efficient, lipid mediated DNA transfection procedure. Proc. Natl. Acad. Sci. USA, 84, 7413-7417.

FRESHNEY, R. 1986. Introduction: principles of sterile techniques and cell propagation. In: Animal Cell Culture: $a$ Practical Approach, IRL Press; Oxford.

GALE, K. R. AND CRAMPTON, I. M. 1989. The ribosomal genes of the mosquito, Aedes aegypti. Eur. J. Biochem., 185, 311-317.

GEHRING, w. J. 1987. Homeoboxes in the study of development. Science, 236, 1245-1252.

GROVER, K. K. 1985. Chemosterilization trials against Aedes aegypti. In: Laird, M. and Miles, J. W. (eds) Integrated Mosquito Control Strategies. Vol. 2, Academic Press, London, pp. 79-116.
HARDIN, P. E., HALL, J. C. AND ROSBASH, M. 1990. Feedback of the Drosophila period gene product on circadian cycling of its messenger RNA levels. Nature, 343, 536-540.

JAMES, A. A., BLACKMER, K. AND RACIOPPI, J. v. 1989. A salivary gland-specific, maltase-like gene of the vector mosquito, Aedes aegypti. Gene, 75, 73-83.

KNIPLING, E. F., LAEVEN, H., CRAIG, G. B., PAL, R., KITZMILlER, B., SMITH, C. N AND BROWN, A. W. A. 1968. Genetic control of insects of public health importance. Bull. WHO, 38, 421-438.

KRAFSUR, E. S., WHITTEN, C. J. AND NOVY, J. E. 1987. Screwworm eradication in North and Central America. Parasitology Today, 3, 131-137.

KUNZE, R. AND STARLINGER, P. 1989. The putative transposase of transposable element Ac from Zea mays $\mathrm{L}$ interacts with sub-terminal sequences of Ac. EMBO J., 8, 3177-3185.

LANGHINRICHS, C., BERNDORFF, D., SEEFELDT, C. AND STAHL, U. 1989. G418 resistance in the yeast Saccharomyces cerevisiae: comparison of the neomycin resistance genes from Tn5 and Tn903. Appl. Microbiol. Biotechnol., 30, 388-394.

LOPATA, M. A., ClEVELAND, D. W. AND SOLLNER-WEBB, B. 1984. High level transient expression of a chloramphenicol acetyl transferase gene by DEAE-dextran mediated DNA transfection coupled with a dimethyl sulfoxide or glycerol shock treatment. Nucl. Acids. Res., 12, 5707-5717.

LYCETT, G. J. 1990. DNA transfection of mosquito cells in culture. Insect Molecular Genetics Newsletter, 4, 1-3. Royal Entomological Society of London Publication.

MACDONALD, W. W. AND RAMACHANDRAN, C. P. 1965. The influence of the gene $f^{m}$ (filarial susceptibility, Brugia malayi) on the susceptibility of Aedes aegypti to seven strains of Brugia, Wuchereria and Dirofilaria. Ann. Trop. Med. Parasitol., 59, 64-73.

MCDONALD, P. T., HAUSERMANN, N. AND LORIMER, N. 1977. Sterility introduced by release of genetically altered males to a domestic population of Aedes aegypti at the Kenya coast. Am.J. Trop. Med. Hyg., 26, 553-561.

MCGRANE, v., CARLSON, J. O., MILLER, B. R. AND BEATTY, B. J. 1988. Microinjection of DNA into Aedes triseriatus ova and detection of integration. Am. J. Trop. Med. Hyg., 39, 502-510.

MERRYWEATHER, A. T., TOWNSON, H. AND CRAMPTON, J. M. 1990. Purification and properties of an esterase from organophosphate resistant Culex quinquefasciatus. Biochem. J., 266, 83-90.

MILLER, L. H., SAKAI, R. K., ROMANS, P., GWADZ, R. W., KANTOFF, P. AND CAON, H. G. 1987. Stable integration and expression of a bacterial gene in the mosquito Anopheles gambiae. Science, 237, 779-781.

MORRIS, A. C., EGGLESTON, P. AND CRAMPTON, J. M. 1989. Genetic transformation of the mosquito Aedes aegypti by microinjection of DNA. Med. Vet. Entomol., 3, 1-7.

MOUCHES, C., PASTEUR, N., BERGE, J. B. et al. 1986. Amplification of an esterase gene is responsible for insecticide resistance in a California Culex mosquito. Science, 233, 778-780.

o'BRochtA, D. A. AND HANDLER, A. M. 1988. Mobility of P elements in drosophilids and non-drosophilids. Proc. Natl. Acad. Sci. USA , 85, 6052-6056. 
POTTER, S. S., BRORIEN, W. J., DUNSMUIR, P. AND RUBIN, G. M. 1979. Transposition of elements of the 412, copia and 297 dispersed repeated gene families in Drosophila. Cell, 17, 429-439.

REDDY, P., ZEHRING, W. A., WHEELER, D. A. et al. 1984. Molecular analysis of the period locus in Drosophila melanogaster and identification of a transcript involved in biological rhythms. Cell, 38, 701-710.

RIO, D. C., BARNES, G., LASKI, F. A., RINE, J. AND RUBIN, G. M. 1988. Evidence for Drosophila $\mathrm{P}$ element transposase activity in mammalian cells and yeast. J. Mol. Biol., 200, 411-415.

RUDNICK, A. 1967. Aedes aegypti and haemorrhagic fever. Bull. WHO, 36, 528-532.

SASA, M. 1976. A Global Survey of Epidemiology and Control. University of Tokyo Press, Tokyo.

SNYDER, M. AND DAVIDSON, N. 1983. Two gene families clustered in a small region of the Drosophila genome. J. Mol. Biol., 166, 101-118.

SPRADLING, A. C. AND RUBIN, G. M. 1982. Transposition of cloned
P elements into Drosophila germ line chromosomes. Science, 218, 341-347.

STELLER, H. AND PIRROTTA, v. 1985. Transposable $\mathbf{P}$ vector that confers selectable G418 resistance to Drosophila larvae. EMBO J., 4, 167-171.

STURCHLER, D. 1989. How much malaria is there Worldwide? Parasitology Today, 5, 39.

WHITTEN, M. J. 1970. Use of chromosome rearrangements for mosquito control. In: International Atomic Energy Agency Symposium, The Sterility Principle for Insect Control or Eradication, IAEA, Athens, pp. 399-410.

WIGLER, M., SIVERSTEIN, S., LEE, L. S., PELLICER, A., CHENG, Y. AND AXEL, R. 1977. Transfer of purified Herpes virus thymidine kinase gene to cultured mouse cells. Cell, 11, 223-232.

WOOD, R. J., COOK, L. M., HAMILTON, A. AND WHITELAW, A. 1977. Transporting the marker gene $r e$ (red eye) into a laboratory cage population of Aedes aegypti (Diptera: Culicidae), using meiotic drive at the $\mathrm{M}^{D}$ locus. J. Med. Entomol., 14, 461-464. 